\title{
Special issue on pervasive and ubiquitous solutions for cultural enrichment
}

\author{
Wei Wei ${ }^{1} \cdot$ Jinsong Wu ${ }^{2} \cdot$ Chunsheng $\mathrm{Zhu}^{3}$ \\ Published online: 13 December 2019 \\ (C) Springer-Verlag London Ltd., part of Springer Nature 2019
}

Cultures and heritages have been preserved in archeological centers, museums, historical centers, art galleries and exhibitions, and cultural artifacts provide rich information and insight about the cultural developments, transformations, traditional and ethics.

Applying pervasive and ubiquitous computing technologies to culture and heritage provide immense benefits but a host of complexities, and this special issue of Personal and Ubiquitous Computing focuses on recent and research the looks at pervasive and ubiquitous solutions for cultural enhancement.

The aim of this special issue is to attract recent research and contributions of high-quality papers in the relevant topics. Based on the feedbacks of peer-reviewers, as well as the evaluations of editors, 33 papers are selected in this special issue from 74 papers. The 33 papers which cover broad topics are selected and briefly introduced as follows.

In the paper "The Construction of Sports Culture Industry Growth Forecast Model Based on Big Data", the feasibility of sports culture industry growth prediction based on big data theory from the perspective of data mining is analyzed, and a sport culture industry growth prediction model based on genetic neural network is proposed. The simulation results demonstrate that the genetic neural network can effectively improve the efficiency and accuracy of prediction and show strong robust in predicting the laws of sports culture industry.

Wei Wei

weiwei@xaut.edu.cn

1 School of Computer Science and Engineering, Xi' an University of Technology, Xi'an 710048, China

2 Department of Electrical Engineering, Universidad de Chile, 833-0072 Santiago, Chile

3 Department of Electrical and Computer Engineering, The University of British Columbia, Vancouver, BC V6T 1Z4, Canada
In the paper "Design on Intelligence Music System in the Cultural Center based on IoT", a set of music intelligent system design scheme in the cultural center based on the IoT technology is proposed, which makes the music realize intelligent and efficient management.

In the paper "Dynamic Pricing Analysis of Redundant Time of Sports Culture Hall Based on Big Data Platform", the game relationship between profit and consumption intention of sports cultural hall is analyzed. A big data platform of the pricing model and using mathematical statistics and support vector machine is constructed. Compared with different pricing strategies, the overall profit of sports cultural hall has been greatly improved.

In the paper "Personalized Recommendation of Film and Television Culture Based on Intelligent Classification Algorithm", a new personalized recommendation algorithm for film and television culture based on intelligent classification algorithm is proposed. The experimental results show that the personalized recommendation algorithm based on intelligent classification algorithm improves the scoring accuracy by 0.15 .

In the paper "Study on Large-scale Crowd Evacuation Method in Cultural Museum Using Mutation Prediction RFID", a large-scale crowd of cultural museum is taken as the studied object, and a large-scale crowd evacuation method based on the mutation theory of RFID is proposed. Through the experiment tests on typical scenes, the evacuation control problems of four groups of people are analyzed and discussed. The results prove that the evacuation method proposed in this paper can provide guidance for crowd evacuation in the cultural museum.

In the paper "An Improved Density-Based Single Sliding Clustering Algorithm for Large Datasets in.

the Cultural Information System", a technique for using a Hybridized Single Sliding Centroid with Density-based Clustering (HSSCDC) to data mine cultural datasets on the cultural information system is introduced. Experimental validation shows that the proposed HSSCDC automatically 
identifies data patterns in CAS web clusters with greater precision, less computational complexity, and less error than previous methods.

In the paper "Basketball Culture Event Detection based on Comprehensive Integrated Method", a comprehensive evaluation model is proposed to solve the defect that the basketball culture weight is difficult to accurately determine. The simulation results show that the model can effectively calculate the correlation coefficient between various factors, and realize the accurate determination of the weight of the basketball cultural factors score index, which provides a reference for the sustainable development of basketball culture.

In the paper "Maximum-Expectation Integrated Agglomerative Nesting Data Mining Model for Cultural Datasets", a MAX-EXP-AN algorithm for locally support clusters or frameworks is designed and developed. The algorithm depends on various parameters that need to be adjusted to achieve optimized parameters for regression, feature selection, and classification. The experimental analysis shows that the proposed algorithm is more suitable for the Cultural Geo Information System (CGIS) to create ubiquitous environments.

In the paper "Intelligent Library Knowledge Innovation Service System Based on Multimedia Technology", the elements and extension mechanism of knowledge service system of intelligent library is analyzed. A multidimensional optimization simulation study of knowledge innovation service quality of intelligent library is constructed. The simulation results verify the validity and advancement.

In the paper "College Public Sports Culture Practice based on Decision Tree Algorithm", the various links that affect the public sports culture of colleges are considered to propose a novel method. Through an example, the method of this paper has been verified, and has certain guiding value for the study of public sports culture.

In the paper "Improvement of Culture Media Efficiency in Internet of Things based on Global Numerical Ant Colony Algorithm", cultural media is taken as the object, the IoT technology is adopted to construct the IoT culture media efficiency optimization model of the global numerical ant colony algorithm. The experimental results show that the global numerical ant colony algorithm based on the IoT is better than the traditional ant colony algorithm.

In the paper "Soft Trees with Neural Components as Image Processing Technique for Archaeological Excavations", a novel soft tree decision structure is proposed. The idea is based on soft sets. In addition, the paper has introduced convolutional networks to the nodes to make decisions based on graphic files. A new archeological item is photographed and evaluated by the proposed technique.

In the paper "Application of Unsupervised TSK Fuzzy Algorithm in Large-scale Online Culture Course", a new mapping method is proposed to effectively improve the learning ability of complex nonlinear data algorithms in TSK fuzzy systems. Results prove the algorithm can effectively identify online culture courses of overlapping scenes without detailed matching process and geometric verification. Compared with the classical fuzzy clustering method, the algorithm has superior and stable performance.

In the paper "Cultural Communication in Double-layer Coupling Social Network based on Association Rules in Big Data", combining the online two-layer coupling network and the CRF word segmentation algorithm, the construction of online social network link prediction is completed. By comparing the information dissemination in social networks and the information dissemination in two-layer coupled social networks, the control of certain lyric cultures can be effectively prevented.

In the paper "Application Analysis of AI Reasoning Engine in Microblog Culture Industry", the artificial intelligence search engine and the classification as well as the characteristics of Weibo data are introduced. Through the test of system performance, it is concluded that the artificial intelligence reasoning system can be well coupled with the microblog culture industry, and will bring users a new humanized experience on the Internet.

In the paper "Design of Sports Culture Data Fusion System based on Data Mining Algorithm", K-means spatial clustering, fusion decision tree, Naive Bayes and other data mining algorithms and data warehouse technologies are combined to the sports stationery industry. An actual case is analyzed using the proposed technology. The results prove the proposed technology can provide a scientific reference model and basis for the modern sports stationery industry.

In the paper "Space-time Distribution Model of Visitor Flow in Tourism Culture Construction via Back Propagation Neural Network Model", the space-time behavior of tourists is studied and analyzed firstly. Finally, a neural network-based prediction model of the space-time distribution of tourists is established. The collected data is brought into the neural network. By comparing the predicted values with the actual values, the model has high prediction accuracy and can be used to predict the spatial and temporal distribution of tourists.

In the paper "Speech Recognition in English Cultural Promotion via Recurrent Neural Network", recurrent neural network and convolutional neural network are used to recognize tone and tone color of English comprehensively, and studies the optimal number of layers and neuron nodes when the recognition accuracy is highest. When the number of layers is 7 and the number of nodes is 256 , the recognition accuracy is the highest, reaching $98 \%$.

In the paper "Research on Music Culture Personalized Recommendation based on Factor Decomposition Machine", the user big data is introduced into the model building process. Through the factor decomposition machine (FM) learning method, the effect of various influencing factors on user behavior is analyzed to build the user dynamic interest model and complete the user preference acquisition. 
In the paper "Multi-level Word Features Based on CNN for Fake News Detection in Cultural Communication", MultiLevel Convolutional Neural Network (MCNN) is proposed, which is introduced the local convolutional features as well as the global semantics features, to effectively capture semantic information from article texts which can be used to classify the new as fake or not. A method of calculating the weight of sensitive words (TFW), which has shown their stronger importance with their fake or true labels. Finally, MCNN-TFW is developed. Extensive experiments have been done on fake news detection in cultural communication to compare MCNN-TFW with several state-of-the-art models, and the experimental results have demonstrated the effectiveness of the proposed model.

In the paper "TouristGo: A Location-based Mobile Game to Improve Tourist Experience by Visiting Path Optimisation", a mobile game TouristGo which not only incentivize the tourists to visit by following the least crowded path but also collect visitors' location data to better manage the tourist flow within the cultural heritage zones is proposed. In addition, through the process, the tourist could gain a better understanding of the knowledge of the cultural heritage zones.

In the paper "Statistical Analysis of Tourist Flow in Tourist Spots Based on Big Data Platform and DA-HKRVM Algorithms", a big data platform based on tourist flow information and a data mining technology based on DA-HKRVM algorithm to predict the tourist flow in the dimension of spatial and temporal distribution are proposed. The simulation result shows that the spatial-temporal distribution model of tourist flow based on data mining has good adaptability and accuracy in application.

In the paper "Database Design of Regional Music Characteristic Culture Resources based on Improved Neural Network in Data Mining", a regional culture and music characteristic resource database is established. Based on data mining technology in combination with the improved BP neural network model, the regional music data in the established database can be handled well.

In the paper "Semantic Change Analysis of Korean Verbs based on Massive Culture Corpus Data", database technology, big data mining and analysis, on-line analytical processing technology and verb feature analysis technology are combined to construct the basic model. Then, the semantic change features of Korean verbs as the object. Finally, the framework of Korean verb semantics feature analysis system based on massive culture corpus data is established. Results prove the designed system has a good application effect.

In the paper "Optimized Integration of Traditional Folk Culture based on DSOM-FCM", a DSOM-FCM model based on dynamic self-organizing neural network is proposed, which integrates resources of traditional folk culture. It not only considers the needs of economic development and spiritual civilization construction, but also meets the needs of social and cultural development. Results show the algorithm designed and used in this paper performs well in solving the optimization and integration of traditional folk culture.

In the paper "Fast Search of Art Culture Resources based on Big Data and Cuckoo Algorithm", a cuckoo search algorithm based on change factors is proposed in the context of big data. The experimental results show that the overall convergence speed of the cuckoo search algorithm based on change factor is obviously better.

In the paper "Logic Detection Method in Network Culture Communication based on Semantic Relevance", based on the convolutional neural network, a detection model of wonderful events is proposed, which achieved the correct detection of goals, corner kicks, penalty kicks and red and yellow cards. It can quickly provide useful cultural information to human beings, enabling such cultures to spread rapidly.

In the paper "Analysis and Data Mining of Intellectual Property Using GRNN and SVM", two kinds.

of mathematical algorithms including GRNN and SVM are introduced to analyze the intellectual property data. By comparing the two kinds of algorithms for intellectual property, the accuracy of GRNN is better than that of SVM in the early state, but it is not better in the later stage. Therefore, it is concluded that the hybrid GRNN and SVM algorithms for intellectual property data mining is much better than the ordinary single algorithm, and it will be widely used in the future data mining data processing.

In the paper "Student Model Construction of Intelligent Teaching System based on Bayesian Network", an intelligent teaching system is built based on Bayesian network. This model can objectively evaluate students' cognitive ability and can infer students' next action. In addition, the model is also applicable to the online test system, and the experimental results obtained demonstrate the effectiveness of the model.

In the paper "Tourism Culture and Demand Forecasting based on BP Neural Network Mining Algorithms", considering the influence degree and quantification difficulty of each factor, the forecast model of tourism demand based on BP neural network is constructed. Through a real example, the proposed model is verified in terms of marketing and pricing strategy, and it puts forward some suggestions for improvement of rural tourism.

In the paper "Exploration on Korean-Chines Collaborative Translation Method based on Recursive Recurrent Neural Network", combining the advantages of the two kinds of neural networks, this paper.

constructs a recursive recurrent neural network model. Based on the tested results, the model can effectively improve the performance of the Korean-Chines Collaborative Translation.

In the paper "An IoT Data Analytics approach for Cultural Heritage", a data analytics approach relying on an Internet of 
Things framework is presented and discussed. The main goal is to assess how the collection of behavioral IoT data coming from the cultural heritage domain can be opportunely exploited by means of data science and data analytics techniques in order to produce useful insights. Experimental results performed in a real case study demonstrate how the cultural heritage domain, and the related stakeholders, can benefit from these kind of applications.
In the paper "Study on Intelligent System of Sports Culture Center with Combining Machine Learning with Big Data", with the help of computer network technology, the design of intelligent system architecture of sports culture center is completed, which makes many intelligent subsystems interconnected, interoperable and information integration, realizes the integration of data application network, and achieves the goal of resource sharing and function upgrading. 